\title{
METAZOÁRIOS PARASITAS DAS NARINAS DO TAMBAQUI Colossoma macropomum (CUVIER, 1818) (CHARACIFORMES: CHARACIDAE) COLETADAS EM LAGOS DE VÁRZEA DA AMAZÔNIA CENTRAL, BRASIL
}

\author{
Germán Augusto M. MOREY ${ }^{1}$, Jose C.O MALTA ${ }^{1}$ \\ 1 Instituto Nacional de Pesquisas da Amazônia, Av. André Araújo, Aleixo, Manaus, Amazonas (germantiss@hotmail.com)
}

\section{RESUMO}

Foram analisadas 32 narinas de tambaqui Colossoma macropomum provenientes de diferentes lagos de várzea da Amazônia Central. Do total de peixes examinados 23 estavam parasitados. Os parasitas identificados foram: Rhinoxenus piranhus (Monogenoidea); Clinostomum marginatum (Digenea); Gamidactylus jaraquensis e Rhinergasilus piranhus (Copepoda); Argulus chicomendensi (Branchiura) e uma sanguessuga (Hirudinea). Os maiores índices de parasitismo foram registrados em Gamidactylus jaraquensis. Este é o primeiro relato da ocorrência de Rhinoxenus piranhus, C. marginatum e Rhinergasilus piranhus parasitando C. macropomum.

PALAVRAS-CHAVE: Tambaqui, parasitos, narinas.

\section{METAZOARY PARASITES OF THE NASSAL FOSSAE OF THE TAMBAQUI, Colossoma macropomum (CUVIER, 1818) (CHARACIFORMES: CHARACIDAE) COLLECTED IN FLOOD PLAIN LAKES OF THE CENTRAL AMAZON, BRAZIL}

\begin{abstract}
Were analyzed 32 nostrils of Tambaqui Colossoma macropomum from different floodplain lakes of Central Amazon. Of the total of fish examined 23 were parasitized. The parasites were identified: Rhinoxenus piranhus (Monogenoidea); Clinostomum marginatum (Digenea); Gamidactylus jaraquensis and Rhinergasilus piranhus (Copepoda); Argulus chicomendensi (Branchiura) and a leech (Hirudinea). The highest parasitism rates were recorded in Gamidactylus jaraquensis. This is the first report of the occurrence of Rhinoxenus piranhus, Clinostomum marginatum and Rhinergasilus piranhus parasitizing C. macropomum.
\end{abstract}

KEYWORDS: Tambaqui, parasites, nostrils. 


\section{INTRODUÇÃO}

O tambaqui Colossoma macropomum (Cuvier, 1818) é uma das espécies mais importantes da ictiofauna amazônica com uma ampla distribuição nas bacias dos rios Orinoco e Amazonas, habitando lagos e áreas alagadas associadas às calhas dos rios principais. Pode alcançar mais de um metro de comprimento e atingir $30 \mathrm{~kg}$. É uma espécie omnívora com marcada preferência por frutas e sementes de alta importância comercial y piscícola na Amazônia (Goulding \& Carvalho, 1982).

A demanda por esta espécie é muito grande e vá aumentando, gerando muito interesse pela sua produção em cativeiro (Varella et al., 2003). Dentro da criança desta espécie existem muitos problemas que deterioram a qualidade do peixe. Desequilíbrios nos parâmetros físico-químicos e inadequado manuseio dos animais quebram o equilíbrio natural entre o parasita e o hospedeiro, dando lugar a epizootias, que podem levar os peixes à morte (Malta et al., 2001).

$\mathrm{Na}$ maioria dos peixes teleósteos as estruturas nasais consistem de um par de cavidades, situado na região ântero-lateral da cabeça, sem nenhuma ligação com a boca. As aberturas externas de cada cavidade são pareadas, por onde flui a água, entrando pela abertura anterior e saindo pela posterior (Romer, 1976).

Para realizar a função olfativa, as fossas nasais devem promover uma continua circulação de água que, por sua vez, pode trazer micro-organismos ou estágios infestantes de parasitas, que rapidamente podem se instalar no epitélio olfativo, causando danos, provocando o aumento na produção de muco, interrompendo o fluxo normal da água, dificultando a olfação, a alimentação e o sentido de orientação dos peixes (Lagler et al., 1977).

Tendo em consideração a importância das narinas como órgão responsável por diferentes atividades fundamentais para os peixes e como hábitat de diferentes organismos, o presente trabalho teve como objetivo principal, o conhecimento da fauna de metazoários parasitas das fossas nasais do tambaqui Colossoma macropomum coletados em lagos de várzea da Amazônia Central.

\section{MATERIAIS E MÉTODOS}

32 tambaquis foram coletados em lagos de várzea da Amazônia Central: cinco lagos do rio Solimões: Baixio $\left(03^{\circ} 17^{\prime} 27,2^{\prime \prime} \mathrm{S} / \quad 60^{\circ} 04^{\prime} 29,6^{\prime \prime} \mathrm{O}\right)$ localizado no município de Iranduba; Preto $\left(03^{\circ} 21^{\prime} 17,1^{\prime \prime} \mathrm{S} / 60^{\circ} 37^{\prime} 28,6^{\prime \prime} \mathrm{O}\right)$ também em Iranduba; Ananá (03⒌ $\left.53^{\prime} 54,8^{\prime \prime} \mathrm{S} / 61^{\circ} 40^{\prime} 18,4^{\prime \prime O}\right)$ em Anori; lago

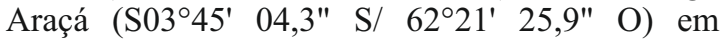

Codajás; lago Maracá $\left(03^{\circ} 50^{\prime} 32,8^{\prime \prime} \mathrm{S} / 62^{\circ} 34^{\prime} 32,4^{\prime \prime O}\right)$ em Coari e um lago do rio Purus, o lago São Tomé $\left(03^{\circ} 49^{\prime} 39,0^{\prime \prime} \mathrm{S} / 6^{\circ} 25^{\prime} 24,6^{\prime \prime} \mathrm{O}\right)$, nos meses de setembro e dezembro de 2013.

Para a captura dos peixes foram utilizadas redes de espera de $20 \mathrm{~m}$ de comprimento por $2 \mathrm{~m}$ de altura com malhas variando de $100 \mathrm{~mm}$ entre nós adjacentes. As redes ficaram na água 10 horas em cada lago no período diurno, com duas despescas.

Os peixes foram identificados, pesados e medidos. As narinas foram retiradas de cada peixe, acondicionadas em recipientes de vidro e fixadas em formol 5\% para posterior análise em laboratório.

As análises foram realizadas no Laboratório de Parasitologia de Peixes do Instituto de Pesquisas da Amazônia (LPP - INPA- Manaus-Brasil). O exame das fossas nasais foi feito conforme a Varella (1992). Lavou-se a cavidade nasal com água destilada. A roseta nasal foi delicadamente retirada do interior do osso nasal, colocando-a em uma placa de Petri com água destilada, lavando-a várias vezes com auxilio de uma pisseta. Examinou-se cada dobra, com auxilio de finos estiletes, coletando-se os parasitas. Observou-se minuciosamente todo o líquido obtido com as lavagens. O líquido dos vidros com as fossas foi decantado e examinado. Todos os exames foram feitos em microscópio estereoscópio.

Os parasitas foram retirados das narinas utilizando estiletes finos e estereoscópio, sendo preservados em formol 5\% (Monogenoidea), AFA (Digenea) e álcool 70\% (Copepoda, Branchiura e Hirudinea). Foram preparadas lâminas permanentes, com montagem total dos parasitas.

Para a identificação dos parasitas as lâminas permanentes foram observadas ao microscópio, com objetiva de 4X,10X, 40X e 100X. Para auxiliar a identificação foram feitos desenhos de cada parasita com o auxílio de câmara clara e ocular micrométrica. A identificação das espécies foi baseada nos trabalhos originais de descrição das espécies.

Os descritores ecológicos do parasitismo (intensidade média, abundância média e prevalência) foram calculados de acordo com Bush et al. (1997) e Serra-Freire (2002). O status comunitário das infracomunidades parasitárias foi classificado segundo Bush \& Holmes (1986): espécies centrais (presentes em mais de dois terços dos hospedeiros), espécies secundárias (em um a dois terços do hospedeiro) e espécies satélites (em menos de um terço do hospedeiro). Foi calculado o Coeficiente de Dominância (CD) de acordo com Serra-Freire (2002), onde o CD mede a porcentagem de uma espécie em relação ao conjunto da infracomunidade parasitária para todos os hospedeiros examinados. 


\section{RESULTADOS}

Os hospedeiros apresentaram um comprimento padrão entre 17 e $30 \mathrm{~cm}$. (média $20 \pm 3,9$ ).

Peso total variando entre 90 e $870 \mathrm{~g}$ (média $307 \pm$ $168,9)$. Foram analisados 32 tambaquis, sendo 23 (72\%) parasitados por pelo menos uma espécie de parasita. Foram encontrados 59 parasitas correspondentes a cinco diferentes grupos: Monogenoidea: Rhinoxenus piranhus Kritsky, Boeger \& Thatcher, 1988 (10,16\%); Digenea: Clinostomum marginatum (Rudolphi, 1819) (1,69\%); Copepoda: Gamidactylus jaraquensis Thatcher \& Boeger, 1984 (79,66\%) e Rhinergasilus piranhus Boeger \&Thatcher, 1988 (5,08\%); Branchiura: Argulus chicomendensi Malta \& Varella, 2000 (1,69\%) e Hirudinea (1,69\%). (Tabela 1).

O comprimento e largura dos parasitas foram registrados, tomando as medidas de todos os indivíduos por espécie coletados. As medidas correspondentes a $R$. piranhus (Monogenoidea); $G$. jaraquensis, $R$. piranhus (Copepoda) são apresentadas em $\mu \mathrm{m}$.

As medidas correspondentes a C. marginatum; A. chicomendensi Malta \& Varella 2000 e Hirudinea são apresentados em mm (Tabela 2).

Tabela 1. Espécies de parasitas com seus respectivos índices parasitários encontrados nas narinas de $C$. macropomum coletados em lagos de várzea da Amazônia Central. PE = Peixes examinados; PP = peixes parasitados; $P(\%)=$ Prevalência; IM $\pm \mathrm{DP}=$ Intensidade Média \pm Desvio padrão; I = Intensidade parasitaria; AM $=$ abundancia media; $\mathrm{CD}=$ Coeficiente de dominância.

\begin{tabular}{lcccccc}
\hline Parâmetros & R. piranhus & C. marginatum & G. jaraquensis & $\begin{array}{c}\text { Rhinergasilus } \\
\text { piranhus }\end{array}$ & A. chicomendesi & Hirudinea \\
\hline PE & 32 & 32 & 32 & 32 & 32 & 32 \\
PP & 1 & 1 & 22 & 3 & 1 & 1 \\
P $(\%)$ & 3,13 & 3,13 & 68,75 & 9,38 & 3,13 & 3,13 \\
IM \pm DP & 6 & 1 & $2,14 \pm 2,2$ & 1 & 1 & 1 \\
I & 6 & 1 & $47(1-9)$ & 3 & 1 & 1 \\
AM & 0,19 & 0,03 & 1,47 & 0,09 & 0,03 & 0,03 \\
CD & 10,17 & 1,69 & 79,66 & 5,08 & 1,69 & 1,69 \\
\hline
\end{tabular}

Tabela 2. Medidas do comprimento e largura das espécies parasitas das narinas de C. macropomum coletados em lagos de várzea da Amazônia Central.

\begin{tabular}{lcc}
\hline \multicolumn{1}{c}{ Espécies } & Comprimento & Largura \\
\hline Rhinoxenus piranhus $(\mu \mathrm{m})$ & $720-925(831)$ & $406-615(497)$ \\
Clinostomum marginatum $(\mathrm{mm})$ & 5,22 & 1,42 \\
Gamidactylus jaraquensis $(\mu \mathrm{m})$ & $420-490(458)$ & $140-203(164)$ \\
Rhinergasilus piranhus $(\mu \mathrm{m})$ & $240-290(270)$ & $99-108(100)$ \\
Argulus chicomendesi $(\mathrm{mm})$ & 4,1 & 2 \\
\hline
\end{tabular}




\section{DISCUSSÃO}

São relatadas para as narinas de C. macropomum duas espécies de metazoários parasitas: o Monogenoidea Notozothecium janauachensis Belmont-Jegú, Domingues \& Martins 2004, registradas por Morais et al., (2009), o Copepoda Gamidactylus jaraquensis Thatcher \& Boeger, 1984 registradas por Fischer et al. (2003) e Perulernaea gamitanae, Thatcher e Paredes, 1985 registrados por Benneton \& Malta, (1999); Fischer et al. (2003). No presente estudo foram encontradas Rhinoxenus piranhus (Monogenoidea), Clinostomum marginatum (Digenea), Gamidactylus jaraquensis e Rhinergasilus piranhus (Copepoda), Argulus chicomendesi (Branchiura) e Hirudinea, sendo todas exceto G. jaraquensis novos registros de parasitas das narinas do tambaqui.

Rhinoxenus piranhus é específico das narinas e as características morfológicas como a presença da âncora dorsal modificada em um esclerito tipo espinho e a ausência de uma barra dorsal estão relacionados com as necessidades de fixação na superfície das narinas (Kritsky et al., 1988). Este parasita foi registrado por estes autores parasitando a cavidade nasal de Pygocentrus nattereri e Serrasalmus altuvei. Morais (2011) encontrou também este parasita nas narinas de $P$. nattereri provenientes de lagos de várzea da Amazônia Central com uma prevalência superior a $70 \%$. Embora $R$. piranhus foi considerado no presente estudo como um parasita raro com baixos índices parasitários já que só foram registrados seis indivíduos em um peixe, este trabalho constitui o primeiro registro de ocorrência deste parasita em $C$. macropomum aumentando assim o numero de hospedeiros.

Quatro espécies de peixes amazônicos foram citadas sendo parasitadas por C. marginatum: Cichla monoculus Spix \& Agassiz, 1831 com metacercarias encistadas nas brânquias; Crenicichla sp. na pele e nadadeiras (Thatcher, 1981); Semaprochilodus insignis (Jardine, 1841) na musculatura (Castelo, 1984) e Pygocentrus nattereri Kner, 1858 com metacercarias na musculatura (Morais et al., 2012). No presente estudo só foi encontrada uma metacercaria de $C$. marginatum nas narinas de $C$. macropomum, sendo este o primeiro registro deste parasita neste hospedeiro. A presença nas narinas pode ser considerada um sinal de alerta para estudos posteriores já que este parasita é considerado com potencial zoonótico associado com a doença conhecida como laringofaringites que pode levar à morte por sufocação (Eiras, 1994).

Rhinergasilus piranhus foi citado parasitando as fossas nasais de $P$. nattereri (Boeger \& Thatcher, 1988; Varella, 1992 e Morais, 2011) indicando a especificidade do parasita tanto pelo hospedeiro quanto para o local de fixação. Embora $R$. piranhus foi considerado um parasita raro com baixos índices parasitários, este estudo constituí o primeiro registro em C. macropomum ampliando assim o número de hospedeiros conhecidos.

Rhinergasilus piranhus é observado usualmente nos seus hospedeiros junto com outros gêneros como Gamidactylus e Gamispatulus (Varella \& Malta, 1995). Os resultados do presente estudo o confirmam já que os três indivíduos de $R$. piranhus registrados foram encontrados juntamente com indivíduos de G.jaraquensis.

Gamidactylus jaraquensis foi descrito pela primeira vez parasitando as fossas nasais de Semaprochilodus insignis no rio Amazonas, próximo a Manaus (Varella, 1992). Fischer et al. (2003), registraram este parasita parasitando as narinas de C. macropomum na região de Coari/Tefé com prevalência parasitaria de $47,2 \%$ e uma intensidade parasitaria variando de $1-6 \mathrm{com}$ uma intensidade média de 3 . No presente estudo este parasita foi o único considerado comum $(\mathrm{P}>50 \%)$ dentro do status comunitário, apresentando uma alta prevalência maior à da Fischer et al. (2003) mas com intensidades médias quase semelhantes a Varella, 1992 e Fischer et al., 2003. Assim mesmo Lacerda et al. (2007) estudando as fossas nasais de peixes da região do Paraná-Brasil, registraram baixos índices parasitários para G. jaraquensis e R. piranhus. Estes resultados indicam que estes parasitas mantém um número baixo de indivíduos em média nos seus hospedeiros, limitados possivelmente pelo pequeno espaço disponível dentro da narina.

O Branchiura Argulus chicomendesi foi registrado por Malta e Varella, (2000) parasitando a superfície do corpo do Tambaqui. No presente estudo só um individuo foi encontrado nas fossas nasais do hospedeiro.

Santos et al. (2013) registrou uma alta prevalência de sanguessugas HirudineaGlossiphonidae parasitando as brânquias de $C$. macropomum. Em peixes o parasitismo branquial ou nas narinas por sanguessugas pode ser acidental (Thatcher 2006; Lemos et al., 2007; Eiras et al., 2010). No presente estudo só uma sanguessuga foi registrada parasitando as narinas de C. macropomum o qual poderia constituir um novo local de fixação do parasita ou um local acidental.

C. macropomum é responsável pela supervivência de sete espécies de parasitas que habitam suas narinas. As espécies encontradas no 
hospedeiro enriquece a biodiversidade amazônica com mais cinco espécies que são pela primeira vez registrada parasitando as narinas deste hospedeiro. Os baixos índices parasitários apresentados no estudo indicam uma possível limitante de colonização ao pequeno espaço disponível dentro da narina.

\section{REFERÊNCIAS BIBLIOGRÁFICAS}

Boeger, W.A.; Thatcher V. E. 1988. "Rhinergasilus piranhus gen. et sp. n.(Copepoda, Poecilostomatoida, Ergasilidae) from the Nasal Cavities of Piranha Caju, Sermsalmus nattereri, in the Central Amazon." Proceedings of the Helminthological Society of Washington, 55 (1), 87-90.

Bush, A.O.; Holmes, J.C. 1986b. Intestinal helmints of lesser scaup ducks: na interactive community. Canadian Journal Zoology, 64: 142-152

Bush, A.O.; Lafferty, K.D.; Lotz, J.M.; Shostak, A.W. 1997. Parasitology meets ecology on its own terms. Journal of Parasitology, 83: 575583.

Castelo, F.P. 1984. Ocorrência de Clinostomum marginatum (Rudolphi, 1819) "Yellow-Spot Disease" em filé de jaraqui (Semaprochilodus insignis Shomburgk, 1814). Acta amazônica, 13, $325-326$.

Eiras, J.C. 1994. Elementos de Ictioparasitologia. Porto: Fundação Engenheiro António de Almeida. 159pp.

Eiras, J.C.; Takemoto, R.M.; Pavanelli, G.C. 2006. Métodos de estudo e técnicas laboratoriais em parasitologia de peixes. $2^{\mathrm{a}} \mathrm{Ed}$. Eduem,Maringá. $199 \mathrm{pp}$.

Fischer, C.; Malta, J.C.O.; Varella, A.M.B. 2003. A fauna de parasitas do Tambaqui, Colossoma macropomum (Cuvier, 1818) (Characiformes: Characidae) do médio rio Solimoes, Estado do Amazonas (AM) e do baixo rio Amazonas, Estado do Pará (PA), e seu potencial como indicadores biológicos. Acta amazônica 33(4): 651-662.

Goulding, M.; Mírian Leal-Carvalho, M. 1982. "Life history and management of the tambaqui (Colossoma macropomum, Characidae): an important Amazonian food fish." Revista Brasileira de Zoologia, 1(2): 107-133.

Kritsky, D. C.; Thatcher, V. E.; Boeger, W. A. 1988. "Neotropical Monogenea. 13. Rhinonastes pseudocapsaloideum n. gen., n. sp.(Dactylogyridae, Ancyrocephalinae), a nasal parasite of curimata, Prochilodus nigricans
Agassiz (Cypriniformes, Prochilodontidae), in Brazil." The Journal of parasitology. 695-698.

Lacerda, A.C.F.; Takemoto, R.M.; Lizama, M.A.P.; Pavanelli, G.C. 2007. Parasitic copepods in the nasal fossae of five fish species (Characiformes) from the upper Paraná River floodplain, Paraná, Brazil. Acta Scientiarum Biological Sciences, 29(4): 429-435.

Lagler, K.F. 1977. Ichthyology. New York: John Wiley \& Sons, 506pp.

Lemos, J.G; Tavares-Dias, M.; Sales, R.S.A., Nobre-Filho, G.R.; Fim, J.D.I. 2007. Parasitos nas brânquias de Brycon amazonicus (Characidae, Bryconinae) cultivados em canais de igarapé do Turumã-Mirim, estado do Amazonas, Brasil. Acta Scientiarum Biological Sciences, 29: 217-222.

Malta, J.C.O.; Varella, A.M.B. 2000. Argulus chicomendesi sp. n. (Crustacea: Argulidae) parasita de peixes da Amazônia brasileira. Acta Amazonica, 30(3): 481-498.

Malta, J.C.O.; Gomes, A.L.S.; Andrade, S.M.S.; Varella, A.M.B. 2001. Infestações maciças por acantocéfalos, Neoechinorhynchus buttnerae Golvan, 1956, (Eoacanthocephala: Neoechinorhynchidae) em tambaquis jovens, Colossoma macropomum (Cuvier, 1818) cultivados na Amazônia central. Acta Amazonica, 31(1): 133-143.

Morais, A.M.; Varella, A.M.B; Correa, M.A.V.; Malta, J.C.O. 2009. A fauna de parasitos em juvenis de tambaqui Colossoma macropomum (Cuvier, 1818) (Characidae: Serrasalminae) criados em tanques-rede em lago de várzea da Amazônia central. Biologia Geral Experimental, 9: 14-23.

Morais, A.M; Varella, A.M.B.; Fernandes, B.M.M.; Malta, J.C.O. 2011. Clinostomum marginatum (Braun, 1899) y Austrodiplostomum compactum (Lutz, 1928) metacercarias con potencial zoonótico en Pygocentrus nattereri (Kner, 1858) (Characiformes: Serrasalmidae) da Amazônia Central, Brasil. Neotropical Helminthology, 5(1): 8-15.

Morais, A.M. 2012. Biodiversidade da piranha vermelha Pygocentrus nattereri (Kner,1858) (Characiformes; Serrasalmidae) e sua avaliação como bioindicadores na Amazônia Central. Teste de doutorado, Manaus, Instituto Nacional de Pesquisas da Amazônia. 243 pp.

Romer, A. S. 1970. Vertebrate Body. 4th Ed. Philadelphia: W. B. Sunders Com., 601pp.

Santos, E.F.; Tavares-Días, M.; Pinheiro, D.A.; Neves, L;R.; Marinho, R.G.B.; Dias, M.K.R. 
2013. Fauna parasitária de tambaqui Colossoma macropomum (Characidae) cultivado em tanque-redeno estado do Amapá, Amazônia oriental. Acta Amazonica, 43(1) 2013: 105-112.

Serra-Freire, N.M. 2002. Planejamento e análise de pesquisas parasitológicas. Editora da Universidade Federal Fluminense, Niteroi, Brasil. 199 pp.

Thatcher, V.E. 1981. Patologia de peixes da Amazônia brasileira, 1. Aspectos gerais. Acta Amazonica, 11, 125-140.

Thatcher, V.E. 2006. Amazon fish parasites. $2^{\mathrm{a}} \mathrm{ed}$. Pensoft Publishe, Sofia, Moscow. 508 pp.

Varella, A.M.B. 1992. Copépodos (Crustacea) parasitas das fossas nasais de peixes, coletados na região de Rondônia, Brasil. Tese de Doutorado, Instituto de Biociências do Campus de Rio Claro, Universidade Estadual Paulista —Júlio de Mesquita Filhol, Rio Claro, São Paulo. 105pp.

Varella, A.M.B; Malta, J.C.O. 1995. Gamydactylus hoplius sp. n. (Copepoda, Poecilomastidea, VAigamidae) das fossas nassais de Hoplias malabaricus (Bloch, 1794) (Characiformes. Erythrynidae) da Amazônia brasileira. Acta Amazônica, 25 (3) 281-288.

Varella, A.M.B; Peiro, S.N; Malta, J.C.O. 2003. Monitoramento da parasitofauna de Colossoma macropomum, cultivado em tanques-rede em um lago de várzea na Amazônia, Brasil. XII Simpósio Brasileiro de Aquicultura, Goiânia, 2: 95-106.

Recibido: 22 de Marzo del 2016

Aceptado para publicación: 25 de Abril del 2016 